\title{
ASSOCIATION BETWEEN PATHOLOGICAL AND MRI FINDINGS IN MULTIPLE
}

\section{SCLEROSIS: AN UPDATE}

${ }^{1,2}$ Massimo Filippi, MD,${ }^{3}$ Wolfgang Brück, MD,${ }^{4}$ Declan Chard, PhD FRCP, ${ }^{5}$ Franz Fazekas, MD,

${ }^{6}$ Jeroen J. Geurts, PhD, ${ }^{5}$ Christian Enzinger, MD, ${ }^{3}$ Simon Hametner, PhD, ${ }^{7}$ Tanja Kuhlmann, MD,

${ }^{1,2}$ Paolo Preziosa, MD ${ }^{8}$ Àlex Rovira, MD,${ }^{9,10}$ Klaus Schmierer, MD,${ }^{3}$ Christine Stadelmann, MD,

${ }^{1,2}$ Maria A. Rocca, MD, for the Attendees of the Correlation between Pathological and MRI

findings in MS workshop*.

${ }^{1}$ Neuroimaging Research Unit and ${ }^{2}$ Dept. of Neurology, Institute of Experimental Neurology, Division of Neuroscience, San Raffaele Scientific Institute, Vita-Salute San Raffaele University, Milan, Italy; ${ }^{3}$ Institute of Neuropathology, University Medical Center, Göttingen, Germany; ${ }^{4}$ Queen Square MS Centre, NMR Research Unit, Dept. of Neuroinflammation, UCL Institute of Neurology, London, UK and National Institute for Health Research (NIHR) University College London Hospitals (UCLH) Biomedical Research Centre, UK; ${ }^{5}$ Dept. of Neurology, Medical University of Graz, Graz, Austria; ${ }^{6}$ Dept. of Anatomy \& Neurosciences, MS Center Amsterdam, Amsterdam Neuroscience, VU University Medical Center, Amsterdam, The Netherlands; ${ }^{7}$ Institute of Neuropathology, University Hospital Münster, Münster, Germany; ${ }^{8}$ Section of Neuroradiology and MRI Unit, Department of Radiology, Hospital Universitari Vall d'Hebron, Universitat Autonoma de Barcelona, Barcelona, Spain; ${ }^{9}$ The Royal London Hospital, Clinical Board Medicine (Neuroscience), Barts Health NHS Trust, London, UK; ${ }^{10}$ The Blizard Institute (Neuroscience), Barts and The London School of Medicine \& Dentistry, Queen Mary University of London, London, UK.

Key words: Multiple Sclerosis; Magnetic Resonance Imaging; Pathology.

Word count: 4874.

References: 127.

Illustrations: 4. Appendix Table, Figure and Panel.

*The full list of participants is given in the Appendix.

Correspondence should be addressed to: Prof. Massimo Filippi, Neuroimaging Research Unit, Institute of Experimental Neurology, Division of Neuroscience, San Raffaele Scientific Institute, Vita-Salute San Raffaele University, Milan, Italy. Telephone number: \#39-02-26433033; Fax number: \#39-02-26435972; E-mail address: filippi.massimo@ hsr.it 


\section{Summary}

Pathological evaluation is the gold standard for identifying multiple sclerosis (MS)-related processes that explain clinical disease manifestations and for guiding the development of new treatments. However, there are intrinsic limitations to the techniques employed, including the limited amount of donors available, samples often representing uncommon cases, and impossibility of follow-up. Correlative studies have demonstrated that MRI is sensitive to the different pathological substrates of MS (inflammation, demyelination and neuro-axonal loss). The role of MRI in evaluating other pathological processes, such as leptomeningeal involvement, central vein and rim of lesions, microstructural abnormalities and iron accumulation as well as recovery mechanisms, has been recently investigated. While techniques used for quantifying pathological processes in different regions of the CNS have advanced the diagnosis and monitoring of MS disease course and treatment, new perspectives and questions have emerged, including how different pathological processes interact over the disease course and when remyelination might occur. Addressing these questions will require longitudinal studies using MRI in large cohorts of patients with different phenotypes. 


\section{Introduction}

Pathological assessment is the gold standard in multiple sclerosis (MS) for understanding the processes involved in the disease and its progression, and for the definition of possible future therapeutic targets. Magnetic resonance imaging (MRI) is a strong partner technology for in vivo evaluation of pathology, due to its high sensitivity in revealing MS-related abnormalities, monitoring disease progression and the effects of treatment. To improve the specificity of MRI in detecting pathological features in MS, association between pathology and MRI needs to be continuously verified. Imaging methods with a high specificity for MS pathology could then be applied longitudinally.

In 2012, a review paper $^{1}$ based on a workshop held by a group of international experts in neuropathology and neuroimaging in MS summarized the evidence from correlative pathologicalMRI studies available at that time. The aim was to discuss concordant findings, but also to highlight controversies, identifying emerging pathological and MRI findings and open questions for future research, which included the need to improve imaging specificity, the acquisition of high-resolution images, the combination of MRI methods and the assessment of specific processes (e.g., remyelination and iron abnormalities). Thanks to the improvement of MRI technologies and larger availability of MRI scanners since 2012, substantial advancements in the field have been obtained. This has allowed better understanding and MRI monitoring of MS-related specific pathological processes, including the presence of central vein sign (CVS) and hypointense rim, the heterogeneous damage in different CNS regions and iron accumulation, but also mechanisms of tissue recovery.

This review provides a summary of the advances in the state-of-the-art of MRI techniques over the past five years and how these correlate to pathological observations. In particular white matter (WM) and gray matter (GM) lesions, normal-appearing brain tissue (NABT) abnormalities, damage of relevant structures (e.g., spinal cord, thalamus, cerebellum, and hippocampus) and iron accumulation were the main topic selected to be discussed during a second workshop (Milan, Italy; 
November 2017), since they represent the main areas of progress in filling the gap between pathology and MRI. Emerging pathological and MRI findings that, in combination, might enhance our understanding of disease pathophysiology and help identify reliable in vivo markers for monitoring different aspects of MS are also presented.

\section{White matter lesions}

Focal WM lesions, characterized by inflammation and demyelination, are the most obvious hallmark of MS histopathology. The inflammatory infiltrate mainly consists of blood-derived monocytes and microglia (for simplicity subsequently termed here as phagocytes), T- and B-cells. ${ }^{2}$ In active and demyelinating lesions, commonly found at the beginning of the disease-as documented in biopsy studies in which the onset of symptoms provides an estimate of lesion development—phagocytes with a round and foamy morphology are the dominating inflammatory cell population (Figure 1). ${ }^{3,4}$ In the very initial stages of lesion formation, these phagocytes contain myelin debris in their cytoplasm suggesting ongoing myelin breakdown as these myelin proteins are degraded within days in vitro by monocytes. ${ }^{4,5}$ During later disease stages, mixed active/inactive lesions with a complete or partial rim of phagocytes and inactive lesions with an almost complete lack of phagocytes become more prominent (Figure 1). ${ }^{3,6}$ The percentage of mixed active/inactive lesions is statistically significantly higher in patients with secondary (SP) and primary progressive (PP) MS compared to relapsing-remitting (RR) MS and correlates with disease severity, ${ }^{3,6}$ therefore MRI correlates that are able to identify this lesions type might be especially valuable to predict the prognosis. The histopathology in early active and demyelinating lesion stages is heterogeneous with respect to the absence or presence of complement depositions, immunoglobulins and oligodendroglial loss, suggesting that different pathological mechanisms may trigger lesion formation. Whether these different histopathological patterns are patient- or lesion-stage specific is still a matter of debate. 
Axonal damage is closely associated with inflammatory demyelination. In the initial lesion stages, axonal spheroids, indicators of transient or permanent axonal transport disturbance and thus axonal damage, are already present. A retrospective study of brain tissue from 39 patients with MS has demonstrated a close correlation between phagocytic infiltration and axonal damage. ${ }^{7}$ The formation of new myelin sheaths around demyelinated axons (remyelination) is frequent in active lesions, and de- and remyelination may occur in parallel within the same lesion. ${ }^{8}$ In a study of 3188 tissue blocks (7562 MS lesions) from the autopsy cohort of the Netherlands Brain Bank, the percentage of remyelinated lesions was higher in RRMS than progressive MS patients. ${ }^{6} \mathrm{~A}$ prerequisite for remyelination is oligodendrocyte progenitor cells that mature to myelinating oligodendrocytes upon demyelination. Whether there is ongoing remyelination in long-lasting MS lesions is a matter of debate; however, results from animal and post mortem studies suggest that remyelination capacity decreases with age and disease duration and that the higher proportion of remyelinated lesions in patients with longer disease duration might be due to a less severe disease. ${ }^{6,9,10}$ Whether the decrease in remyelination capacity is due to decreased activity of phagocytes or to age-associated intrinsic changes in oligodendrocytes has yet to be determined.,11 However, remyelination is relatively infrequently observed in MS patients with a disease duration of more than 10 years. Only about $20 \%$ of the lesions are completely remyelinated (so-called shadow plaques), whereas in the vast majority of lesions, remyelination is either absent or limited to a small rim at the lesion border (Figure 1). ${ }^{12}$

T2-weighted (including fluid-attenuated inversion recovery [FLAIR]) imaging is highly sensitive in detecting focal WM MS lesions, enabling a prompt and accurate diagnosis of MS in patients presenting with clinical syndromes typical of demyelination. However, overreliance on MRI, particularly in the context of atypical clinical symptoms, frequently leads to overdiagnosis of MS, mainly due to a lack of a careful exclusion of alternative diagnoses ${ }^{13}$ and an inappropriate application of diagnostic criteria to define dissemination in space and time. ${ }^{14}$ 
The association between focal MS lesions and venules can now be visualized (the CVS) using susceptibility-based MRI sequences, taking advantage of the $\mathrm{T}_{2}{ }^{*}$-shortening by deoxyhemoglobin in venous blood, particularly when using high magnetic field strength scanners ( $\geq 3 \mathrm{~T}) .{ }^{15}$ The CVS has been proposed as a new MRI biomarker to improve diagnostic accuracy, since its presence in WM lesions can help to differentiate MS from other diseases (Figure 2). ${ }^{16-19}$ CVS conspicuity can be improved by acquiring a susceptibility-weighted sequence after gadolinium administration, particularly at standard clinical magnetic field strengths $(1.5-3 \mathrm{~T}){ }^{20-22}$

Using susceptibility-based MRI at 7T, a hypointense rim is seen in some WM MS lesions (Figure 2). This feature is not present in people with cerebro-vascular disorders, CNS inflammatory vasculopathies or neuromyelitis optica (NMO) $)^{17,18,21}$ and might represent a more specific pathological hallmark of MS. Future studies may demonstrate that incorporating this imaging feature at standard field strengths further improves the specificity of MRI in MS diagnosis. This hypointense rim likely reflects iron accumulation within a subset of macrophages/activated microglia at the edge of chronic active WM MS lesions (MRI reflection of the pathologically defined "mixed active/inactive lesions, see also the iron pathology section), ${ }^{2,22,23}$ although other relevant factors, such as oxidative stress and disruption of tissue microstructural organization may contribute to this signal change in active lesions. ${ }^{24,25}$ On conventional MRI at ultra-high field (7T) in 10 patients with MS (7 in vivo, 4 scanned before pathology), these chronic active lesions were characterized by progressive lowering of $\mathrm{T}_{1}$ signal intensity, and in some cases by a slow increase in size (slowly expanding lesions) (Figure 2) ${ }^{23}$ After a period of time (months to years), these lesions may become inactive, with no macrophage/microglia rim at the lesion border, as shown in a retrospective study of 32 patients with $\mathrm{MS} .^{24}$ Therefore, lesions with hypointense rims have potential as predictors of ongoing inflammation and tissue injury, which is particularly relevant in progressive MS patients, where detection of inflammatory disease activity may help to identify patients most likely to respond to anti-inflammatory treatments and would be highly valuable to improve prognosis since these lesions correlate with severity of MS. ${ }^{3,6}$ Despite this, further 
longitudinal studies are still necessary to evaluate the natural history of hypointense rims. The appendix describes atypical forms of demyelination (tumefactive demyelinating lesions).

\section{Gray matter lesions}

The extent of GM demyelination in MS varies considerably between patients and can be widespread, especially in the chronic phase. In the neocortex, the proportion of tissue affected by demyelination ranges between $15 \%$ and $40 \%$ on histological inspection, while an average of $30 \%$ demyelination is seen for the deep GM, mesencephalon, hippocampus, cerebellar cortex and spinal cord GM, with outliers of near-total demyelination of the GM involved (Appendix). ${ }^{26-33}$

GM lesions are classified according to their location. ${ }^{34}$ This includes mixed GM/WM lesions, where the inflammatory profile of the WM portion resembles that in WM lesions; and the more common subpial lesions, which may extend over several gyri and are generally conspicuously non-inflammatory at autopsy, although representative samples of biopsied MS cortical material from 53 MS patients with GM lesions (a total of 104 lesions) showed extensive involvement of the innate and adaptive immune system (CD3+ T-cell infiltrates and macrophage-associated demyelination). ${ }^{35}$ This discrepancy may be because they have been examined in different phases of the evolution of such lesions. Inflammation is likely to be a key driver of cortical demyelination, since the involvement of C-C chemokine receptor 2 (CCR2)-positive monocytes, as well as B- and T-cells was shown in the cortical GM and overlying meninges, ${ }^{36,37}$ as well as cortical complement deposition ${ }^{38}$ and subtle blood-brain barrier disruption. ${ }^{39}$ Rims of activated microglia were found in autopsy cases of 22 patients with a more aggressive disease course (ie, extensive subpial demyelination) compared with 19 patients with scarce demyelination of the cerebral cortex. ${ }^{40}$ In another study of 27 patients with MS compared with 14 healthy controls, both histopathology and positron emission tomography (PET) showing microglia activation throughout the MS cortex, ${ }^{41}$ increasing in severity with disease progression. The way in which patients respond to damage due to inflammation may be determined by their genetic background. In a study of 47 patients with MS, 
HLA-DRB1*1501-positive individuals expressed more CD3+ and iba1+ microglial reactivity in their cortical lesions (CL), ${ }^{42}$ and in a post mortem study of Netherlands Brain Bank tissue from 12 patients with MS, it appears likely that genetic and epigenetic factors involved in modulating the severity of WM lesions also play a role in the GM. ${ }^{43}$

Using post-contrast FLAIR at 3T in 299 patients with MS, focal leptomeningeal enhancement was found in $74(25 \%)$ cases, with a higher frequency in progressive (39 [33\%] of 188) than RRMS (35 [19\%] of 181) phenotypes. ${ }^{44}$ Subsequent pathological evaluation of two of the cases showed perivascular lymphocytic, mononuclear infiltration and subpial demyelination in enhancing areas. ${ }^{44}$ However, leptomeningeal enhancement is not specific to MS, and was reported to occur with a high frequency in $18(35 \%)$ of 51 patients with other non-MS inflammatory neurological conditions. ${ }^{45}$

Several other pathogenic mechanisms have been recently detected in GM lesions. Damage to neuropilema (which refers to the complex network of unmyelinated axons, dendrites, and glial cells) has been investigated. Atrophy of the cortical GM, measured using MRI, was predominantly determined by neuronal shrinkage and axonal degeneration. ${ }^{46}$ However, neuronal loss, ${ }^{29}$ damage ${ }^{47}$ and spine loss coupled to dendritic degeneration ${ }^{48}$ were also found. The extent to which these changes are related to, or dependent on, inflammation, and their role in explaining clinical symptoms remains a subject of future investigation. ${ }^{49}$

Focal CLs are poorly visualized using conventional MRI sequences (including dual-echo $\mathrm{T}_{2}$ weighted and FLAIR) due to their small size, poor contrast with the surrounding normal appearing (NA) GM, and partial volume effects with WM and cerebrospinal fluid (CSF). Specialized sequences, such as double inversion recovery, ${ }^{50}$ phase-sensitive inversion recovery ${ }^{51}$ and threedimensional magnetization-prepared rapid acquisition with gradient echo ${ }^{52}$ have improved our ability to detect GM lesions. However, even a technique such as 3D double inversion recovery only had a sensitivity of $18 \%$ to detect CLs in a post mortem study of 56 matched brain samples from 14 patients with $\mathrm{MS},{ }^{53}$ with a relatively low inter-observer consensus (19.4\% of all CLs detected) ${ }^{50}$ 
The use of ultra-high field scanners has improved the identification of CLs, ${ }^{54}$ allowing to describe lesion patterns that resemble those reported by pathology. ${ }^{55}$

CL evaluation is important in the diagnostic work-up of suspected cases of MS. In patients with a clinically isolated syndrome (CIS), the presence of a single CL identifies those subjects almost certain to have MS confirmed later. ${ }^{56} \mathrm{CLs}$ have not been seen, so far, in other conditions that can mimic MS, including migraine ${ }^{57}$ and NMO. ${ }^{58}$ Therefore, adding them to diagnostic criteria is likely to improve their specificity. ${ }^{59} \mathrm{CLs}$ have been shown in all MS phenotypes, especially in the progressive forms, and the number and volume of CLs correlated with disability and cognitive impairment, as well as with worsening of disability and clinical phenotype after two, five, and seven years of follow-up (Appendix). ${ }^{60}$

Using high- and ultra-high field scanners, a correlation between reduction of magnetization transfer ratio (MTR) values and the presence of focal cortical demyelination has been reported, suggesting that MTR is sensitive to cortical demyelination/remyelination processes. ${ }^{61,62}$ In vivo, using clinical MRI scanners, MTR reduction in the outer cortical surface has been detected in MS patients with the main phenotypes (i.e., RRMS, SPMS and PPMS), with the lowest values seen in SPMS. ${ }^{63,64}$ Abnormal MTR values were preferentially located in the cingulate cortex, insula, and the depths of sulci, in agreement with pathological descriptions of subpial demyelination location. ${ }^{63}$

Using diffusion tensor (DT) MRI, it has been shown that, unlike WM lesions (which usually show decreased fractional anisotropy [FA]), CLs have an increased FA compared to NAGM, ${ }^{65,66}$ while conflicting results have been found for mean diffusivity (MD). ${ }^{65-68}$ Different pathological substrates have been proposed to explain these diffusivity abnormalities, including damage to and loss of synapses, dendrites and cortical neurons. Neuroaxonal loss/shrinkage has been suggested by a study of 26 patients with MS and 24 healthy controls which applied neurite orientation dispersion and density imaging (NODDI) and showed a decreased intracellular volume fraction. ${ }^{67}$ A recent $7 \mathrm{~T}$ correlative MRI/pathology study of 14 patients showed higher cell density in CLs, with few 
activated microglial cells or increased cell size,${ }^{69}$ suggesting increased cellular density and tissue compaction as a possible mechanism for CL diffusivity changes.

The quantification of $T_{1} / T_{2}$ ratio (the ratio of $T_{1}$ - to $T_{2}$-weighted image intensities) has been proposed as a possible biomarker of myelin content and cortical integrity. Decreased $\mathrm{T}_{1} / \mathrm{T}_{2}$ ratio (which correlated with MTR reduction) was found in demyelinated cortex compared to NAGM of 6 post mortem MS brains. ${ }^{70}$ The specificity of this technique for cortical demyelination needs further validation since another post mortem study of 9 brains from patients with MS reported a correlation between decreased cortical $\mathrm{T}_{1} / \mathrm{T}_{2}$ ratio and dendrite density (but not myelin content). ${ }^{71}$

\section{Spinal cord}

Spinal cord pathology is common in MS and, compared to the brain, more likely to be clinically apparent. Acute symptoms and signs at onset and the chronic loss of function, particularly of the lower limbs, correlate to some extent with findings in pathology specimens where blood brain barrier damage, inflammation, demyelination, gliosis and neuro-axonal loss, have been observed..$^{30,72,73}$

A post mortem study of 13 people with MS and 5 non-neurological controls that examined the extent and location of demyelination across different cord levels showed more extensive demyelination in GM (24-48\%) than WM (11-13\%), and in the thoracic level compared to other segments of the spinal cord. ${ }^{30}$ Depicting and quantifying cord pathology in vivo using MRI remains a challenge. At high field (4.7T) using post mortem spinal cord tissue from 11 patients with MS and two non-neurological controls, 53 (87\%) of 61 of WM lesions and $32(73 \%)$ of 44 GM lesions were detected. ${ }^{74}$ While replicating this sensitivity in vivo is not yet achievable, the presence of cord lesions is incorporated into current MRI diagnostic criteria for MS. ${ }^{75}$ Cervical cord cross sectional area (CSA) shrinkage has been shown to correlate with motor disability in a study of 335 patients with CIS or MS compared with 143 healthy controls, ${ }^{76}$ and has been used in a small number of clinical trials. ${ }^{77-79}$ 
There is growing interest in more tissue-specific MRI measures, and a study of 159 patients with MS has shown that it is possible to assess spinal cord GM and WM atrophy separately. ${ }^{80}$ This methodological advance coincided with pathology data indicating that cord CSA does not predict cortico-spinal tract axonal loss, ${ }^{30}$ a surprising finding given the long-held belief that axonal loss is the major correlate of chronic disability (Figure 3). One possible explanation for these conflicting results may be the loss of synapses, which preliminary data suggests may outweigh the loss of long tract axons, and indeed seems to correlate with spinal cord GM CSA, a measure that has been shown to predict disability better than the total cord CSA in 113 patients with MS compared with 20 healthy controls. ${ }^{81}$

Techniques that measure the size or volume of segmented tissue compartments may for the foreseeable future remain the most commonly used "translators" between tissue microstructure and clinical indices. However, substantial strides are also being made with techniques that try to quantify microstructure in the spinal cord. These include short $\mathrm{T}_{2}$ relaxation, MTR, DTI and postprocessing algorithms, with a shift in focus from measures of myelination, ${ }^{82}$ to measures of axons and neurites. Two new diffusion-based techniques, diffusion basis spectrum imaging (DBSI) ${ }^{83,84}$ and NODDI ${ }^{85-87}$ appear promising. Both DBSI and NODDI have been applied in vivo and to human post mortem spinal cord showing strong correlations between the quantitative histology and MRIderived measures of microstructural complexity. ${ }^{83-87}$

\section{Normal-appearing brain tissue}

Pathologically, NAWM has been defined as macroscopically normal WM that is microscopically normally myelinated, is at least $10 \mathrm{~mm}$ away from a plaque's edge, and is differentiated from diffusely abnormal or dirty-appearing WM, including areas of diffuse myelin pallor with ill-defined borders. ${ }^{88} \mathrm{NAGM}$ has been defined as a region with no evidence of demyelination using immunohistochemistry. ${ }^{34}$ Regarding MRI, the definitions of NABT depend on 
the sensitivity and specificity in the detection of tissue alterations and are prone to changes with technical advances. $^{89}$

A post mortem in situ MRI study, including magnetization transfer (MT) MRI and DT MRI, assessed the pathological basis of subtle NAWM abnormalities in four SPMS brains. ${ }^{90}$ MT MRI and DT MRI metrics correlated moderately with myelin density, axonal area, and axonal counts. In “truly” NAWM (defined as WM tissue without $\mathrm{T}_{2}$-hyperintense, $\mathrm{T}_{1}$-hypointense, and MTR-visible "lesions"), MTR and DT MRI measures only correlated with activated microglia, ${ }^{90}$ indicating that pathological changes in MS brains may be present even in "true" NAWM as defined by both conventional and non-conventional MRI, such as MT and DT MRI. A 7T MR-PET imaging study with [11C]-PBR28 (18kDa translocator protein; TSPO; a marker of activated microglia/macrophages) provided in vivo evidence of widespread microglial activation in the GM of 12 RRMS and 15 SPMS brains. ${ }^{41}$ Relative to 14 healthy controls, patients exhibited abnormally high [11C]-PBR28 binding, predominantly in cortex and CLs, thalamus, hippocampus, but also NAWM, while focal WM lesions showed only modest increases. Cortical thinning correlated with increased thalamic TSPO levels, ${ }^{41}$ suggesting widespread neuroinflammation partially linked to neurodegeneration.

The hypothesis that common CSF-mediated factors might contribute in the accumulation of GM and WM damage has been tested in 160 patients with MS, via correlations between increased periventricular $\mathrm{T}_{2}$-lesion burden and decreased cortical thickness, indicative of subpial pathology. ${ }^{91}$ Compared to 58 healthy controls, 91 patients with CIS and 69 with RRMS had reduced cortical thickness; the reduction was even more pronounced in RRMS patients than in ones with CIS. Increased periventricular lesion occupancy correlated with decreased cortical thickness in RRMS, but not in CIS. ${ }^{91}$ Using MT MRI, ${ }^{92}$ proximity to the ventricular surfaces was associated with progressively lower MTR values in 67 patients with relapse-onset MS in supratentorial and cerebellar NAWM, brainstem, and deep and cortical GM compared with 30 healthy controls, providing evidence for common factors underlying the spatial distribution of microstructural 
abnormalities in NAWM and GM. A gradient in cortical pathology throughout different MS phenotypes has also been observed at $7 \mathrm{~T}$ using surface-based analysis of $\mathrm{T}_{2}{ }^{*}$ relaxation rates. ${ }^{93}$ Relative to 17 healthy controls, 41 patients with CIS or MS demonstrated increased $\mathrm{T}_{2}{ }^{*}($ consistent with cortical myelin and iron loss), independent from cortical thickness. In early disease, $\mathrm{T}_{2}{ }^{*}$ changes were focal and mainly confined to the first $25 \%$ of depth and to cortical sulci. In later stages, $\mathrm{T}_{2}{ }^{*}$ changes involved deeper cortical laminae, multiple cortical areas and gyri.

Longitudinal studies with advanced MRI to assess NABT changes in MS are scarce. One study in 11 patients with RRMS and 4 healthy controls looked at measures sensitive to myelin content and intra/extracellular water properties in RRMS NAWM at baseline and 3.2-5.8 years and found progressive changes in myelin integrity occurring diffusely. ${ }^{94}$

\section{Thalamus, cerebellum and hippocampus}

Pathological and MRI studies have consistently demonstrated an early involvement of the thalamus, cerebellum and hippocampus. Damage of these structures is clinically relevant, since it is strongly associated with locomotor disability and cognitive impairment. ${ }^{95-97}$ Other clinically relevant structures are represented by the brainstem and hypothalamus, however they have not been discussed during the workshop, and are therefore outside the scope of this Review.

The thalamus may show considerable demyelination in progressive disease. ${ }^{95}$ In a study of 14 brains from patients with MS and 12 controls (6 from non-neurological controls, 6 from patients with amyotrophic lateral sclerosis), demyelination solely in GM and mixed GM-WM lesions occurred particularly in the periventricular medial and anterior thalamic nuclei, but not in the ventral thalamic nuclei. ${ }^{27}$ Thalamic neuronal loss and neuronal atrophy can be substantial, affecting demyelinated and non-demyelinated tissue areas equally. ${ }^{27,98}$ In 14 patients with SPMS compared with 14 non-neurological controls, a 30-35\% reduction in neuronal density was found in the medial dorsal nucleus, corresponding to a $22 \%$ reduction in volume by MRI, and a $19 \%$ reduction in Nacetylaspartate. ${ }^{98}$ Microglia activation was not restricted to demyelinated areas, but also occurred in 
non-lesioned thalamus. Inflammatory infiltration was in general lower in GM compared to WM regions. $^{27}$

Cerebellar symptoms, including Charcot's triad of nystagmus, intentional tremor and dysarthria, are frequent in MS. On average 38\% of the cerebellar cortex has been found to be demyelinated across several studies. ${ }^{26,31}$ In these studies, lesions were often purely cortical, but also mixed GM/WM lesions were found. Cerebellar cortical demyelination has also been associated with subarachnoid inflammation. ${ }^{32}$ Neuronal pathology has also been detected, with a slight reduction in Purkinje cell density, especially in demyelinated areas. ${ }^{32,99}$ Neuronal atrophy and loss, as well as synaptic reduction have also been observed in the deep cerebellar nuclei, irrespective of the presence of focal demyelinated lesions. ${ }^{33}$

Demyelinated hippocampal lesions have been detected in the majority of patients with chronic MS, with mixed hippocampal and parahippocampal lesions being frequent. In one study of tissue from 19 patients with MS compared with 7 non-neurological controls, ${ }^{28}$ the hilus and cornu ammonis (CA) 2 region were only affected if large areas were demyelinated, but not in isolation. In 9 of 19 affected patients, cognitive decline had been noted in the patients' medical files before autopsy. ${ }^{28}$ In a study with 45 patients with progressive MS and 7 non-neurological controls, 22 (54.3\%) of 41 lesions found in MS patients were partially contained in the subependymal or subpial regions. ${ }^{100}$ Lesions were mainly present in the CA1 region, whereas CA4 was only rarely demyelinated. ${ }^{100}$ A statistically significant reduction (46\%) of synaptic density was observed in CA4 of MS patients. ${ }^{100}$ Synaptic loss outweighed neuronal reduction and was independent of focal demyelination. ${ }^{100}$ In another post mortem study of tissue samples from 15 patients with MS compared with 10 non-neurological controls, molecular alterations in hippocampal neurotransmission accompanied neuroaxonal loss. ${ }^{101}$

Using MRI, focal lesions have been consistently seen in the thalamus, cerebellum and hippocampus of the main MS phenotypes. ${ }^{95-97}$ Assessing the presence and extent of lesions in these critical structures has a clinical role not only for diagnosis (e.g., the cerebellum is one of the typical 
locations for demonstrating disease dissemination in space), ${ }^{75}$ but also for improving understanding of some of disease symptoms, including cognitive deficits ${ }^{102}$ and motor impairment. ${ }^{103}$

Quantitative MR techniques have shown microstructural abnormalities beyond focal lesions, which are more severe in progressive MS patients. ${ }^{103-105}$ Such microscopic abnormalities have even been observed to occur in CIS patients. ${ }^{106}$ Correlative studies have reinforced the clinical relevance of microscopic tissue alterations in these regions. For example, in a study of 24 patients with MS compared with 24 healthy controls, increased thalamic MD correlated with motor dysfunction, particularly in the 11 patients with RRMS, ${ }^{104}$ whereas in 54 patients with PPMS compared with 8 healthy controls, decreased thalamic FA predicted worsening of disability after five years. ${ }^{107}$ In 37 patients with CIS, increased hippocampal MD distinguished cognitively impaired from cognitively preserved participants. ${ }^{106}$

Atrophy also occurs in the thalamus, cerebellum, and hippocampus already from the very early temporal stages of the disease (i.e., CIS and pediatric MS), thus in patients with a shorter time between the clinical and the biological onset of the disease. Consistent with the regional susceptibility to damage of the main hippocampal subfields (CA1 to CA4, dentate gyrus and subiculum), regional analyses have shown an uneven distribution of atrophy in the hippocampus, with more severe involvement of the CA1 and subiculum than other hippocampal subfields ${ }^{108,109}$ In a study of 95 patients with MS compared with 32 healthy controls, an association between the functional specialization of the subregions of the cerebellum and clinical measures was observed, with volume of the posterior cerebellum accounting for variance in cognitive measures, while anterior cerebellar volume accounted for variance in motor performance. ${ }^{110} \mathrm{~A}$ key remaining question is: what drives microstructural and volumetric abnormalities in these structures? Most studies have found a correlation between such abnormalities and WM focal lesion volumes, suggesting secondary degeneration as the cause. A combined DT MRI and atrophy study of 52 patients with MS and 57 healthy controls examined the relative contributions of thalamic damage and its WM connections in explaining cognitive impairment in MS; in the study, damage to the 
cortico-thalamic WM was identified as the most important contributor to cognitive dysfunction. ${ }^{111}$ Correlations were also seen between WM tract abnormalities and thalamic alterations, suggesting a disconnection syndrome. The hypothesis that some of the clinical symptoms attributable to involvement of the thalamus, cerebellum and hippocampus is due to disconnection of these GM structures from integrated functional and structural systems is also supported by functional and DT MRI studies. ${ }^{112-114}$

\section{Iron pathology}

The notion of abnormal brain iron distribution in MS dates back more than 35 years with the observation of iron accumulation around demyelinated MS plaques, using the Perls' staining for ferric iron. ${ }^{115}$ Subsequent studies showed decreased signal intensity in several deep GM structures correlated with the amount of iron indicated by Perls' staining in healthy post mortem brains, ${ }^{116}$ and decreased signal intensity on $\mathrm{T}_{2}$-weighted images was seen in the putamen and thalamus of MS patients. ${ }^{117}$ More pronounced $\mathrm{T}_{2}$-hypointensity of the deep GM structures was confirmed in other MS cohorts, and the degree of $\mathrm{T}_{2}$ signal loss was associated with brain atrophy and predictive of disability and clinical course. ${ }^{118}$ Interest in these findings was revived by two avenues of research. New MRI techniques such as $\mathrm{R}_{2}{ }^{*}$ mapping ${ }^{119}$ (Figure 4) and quantitative susceptibility mapping ${ }^{120}$ have been developed and have been validated in post mortem studies. ${ }^{119-121}$ In addition, it was suggested that iron liberated from destroyed cells promotes neurodegeneration by enhancing chronic oxidative injury. ${ }^{122,123}$ Subsequent MRI and pathology research have followed somewhat different directions to clarify the role of iron in MS.

Quantitative MRI studies have concentrated primarily on the assessment of iron in the deep GM because of the confounding effects of myelin in WM, and signal contamination from adjacent tissues when examining the cortex. Age of the patient has also to be considered, since iron normally accumulates in the deep GM at least during the first four decades of life. In a cross-sectional multicentre setting of 97 patients with MS compared with 81 healthy controls, $\mathrm{T}_{2}{ }^{*}$ values in the 
basal ganglia, indicative of iron concentration, increased from the stage of CIS, through RR to progressive MS, ${ }^{124}$ while a 2-year longitudinal study of 17 patients with MS compared with 17 healthy controls showed an increase in $\mathrm{R}_{2}{ }^{*}$ (the inverse of $\mathrm{T}_{2}{ }^{*}$ ) in the basal ganglia that was related to disability. ${ }^{125}$ These findings were confirmed by a 3-year longitudinal study, that documented an increase in $\mathrm{R}_{2}{ }^{*}$ in the basal ganglia, which was more pronounced in 76 patients with CIS than in 68 MS patients (62 RRMS and 6 SPMS). ${ }^{126}$ A reduction in magnetic susceptibility, presumably related to decreased iron concentration of the thalamus, was also reported in a study of 140 patients with CIS or MS, which may be a consequence of iron depletion from oligodendrocytes. ${ }^{127}$ Pathological confirmation of all these observations is still needed.

In a histopathological study of 4 patients with MS and 3 non-neurological controls, iron concentration in the NAWM of MS patients decreased statistically significantly with disease duration, in contrast to the physiologic increase seen in controls with aging. ${ }^{122}$ In active MS lesions, iron is apparently released from dying oligodendrocytes, but accumulates in activated microglia and macrophages at the lesion edges, and in astrocytes in inactive centres of a subset of slowlyexpanding lesions. ${ }^{23,122}$ Quantitative correlative assessments in 4 brains of patients with MS confirmed that black rims around lesions on 7T susceptibility-weighted imaging are a consequence of iron-laden microglia/macrophages. ${ }^{23}$ Annual MRI follow-up of 7 patients over 3.5 years showed that the size of lesions with a hypointense rim increased over time which, together with the pathological findings, ${ }^{23}$ led to the suggestion that iron rings might characterize slowly expanding lesions. ${ }^{122}$ In another study of autopsy tissue from 18 patients with MS using synchrotron X-ray fluorescence imaging, iron accumulation in microglia/macrophages at the edge of a subset of slowly-expanding lesions was shown. ${ }^{124}$ However, iron accumulation in astrocytes-primarily in inactive centres of slowly expanding lesions-and in a concentric pattern in a single inactive lesion was also detected, suggesting a possible protective role of astrocytes by capturing and neutralizing extracellular iron. ${ }^{128}$ Iron decreased with patient age in NAWM, cortex and MS lesions, and iron concentration in the periplaque WM was statistically significantly lower than in NAWM. 
Iron in the NAGM and CLs of MS was characterized in a correlative pathology-MRI study of 2 patients with MS, where concomitant loss of iron and myelin was described in CLs relative to surrounding NAGM, and was also observed in the corresponding $7 \mathrm{~T} \mathrm{R}_{2}{ }^{*}$ images. ${ }^{129}$ Another form of focal iron accumulation is in so-called microbleeds, which indicate extravasation of blood through leaking blood vessels. A case control MRI study of 445 patients found cerebral microbleeds in $19.8 \%$ of MS patients $\geq 50$ years compared to $7.4 \%$ in 177 healthy controls. ${ }^{130}$

Brain iron in MS still needs to be explored further. Pathological confirmation of the MRI findings of diffuse changes in the basal ganglia and thalamus are needed. In the WM and lesions, the confounding influence of myelin on $\mathrm{T}_{2}{ }^{*}$ needs to be addressed. One possibility is temperaturedependent relaxometry, but this can only be applied to post mortem tissue. ${ }^{131}$ Longitudinal studies will be needed to determine whether iron accumulation is a trigger of neurodegeneration and propagates lesion expansion or is just a marker of disease progression.

\section{Conclusions and future directions}

Over the past few years, there have been many advances in MRI measures that reflect pathology findings in MS. Among them, the evaluation of leptomeningeal enhancement and of lesional CVS have been proposed to improve the diagnostic work-up of patients with suspected MS. The assessment of microstructural abnormalities and iron accumulation have increased our understanding of the mechanisms responsible for MS clinical manifestations. New methods (e.g., NODDI) to assess pathological processes more specifically and to evaluate tissue recovery (e.g., remyelination) in vivo by means of MRI have also been used in preliminary studies.

Advances in the field have also resulted in new questions, which will stimulate further research. Determining timing and duration of the potential for remyelination in different CNS structures is of crucial importance, since promoting remyelination is one of the main objectives of current treatments. Pathologists and imaging experts should work together to develop and standardize techniques capable of assessing remyelination. Since low-grade inflammation may be 
present at the border of some lesions in selected patients, there is a need to identify pathological, imaging and clinical features of these lesions, to move towards individualized treatment. It will be a challenge to determine the interplay between the different pathological processes (e.g., inflammation, demyelination and neurodegeneration) over time, which calls for longitudinal evaluation using complementary MRI techniques.

Future research should focus on the early stages of the disease, include cohorts with clinically representative cases of different phenotypes, and seek causative information via repeated imaging. This would also allow the focus of the field to be switched from studies seeking to correlate MRI measures with clinical variables to those measures that can predict disease progression. Finally, to establish research priorities and surrogate markers of specific pathological processes (e.g., inflammation, demyelination and neurodegeneration), better communication and collaboration between pathologists and MRI researchers is needed, including direct pathology-MRI correlation close to autopsy, with the acquisition of MRI sequences with brain and spinal cord in situ and within a short time period post mortem. 


\section{Search strategy and selection criteria}

References for this Review were identified through searches of PubMed with the search terms “Autopsy”, "Histopathology”, "Multiple Sclerosis", "MRI”, "Pathology”, "Post Mortem”, “Lesions”, “Normal-Appearing White Matter”, “Gray Matter”, “Spinal Cord”, “Cerebellum”, “Hippocampus”, “Thalamus" and "Iron” from Jan 1, 2013 to Oct 31, 2018. Articles were also identified through searches of the authors' own files. Only papers published in English were reviewed. The final reference list was generated on the basis of originality and relevance to the broad scope of this Review.

\section{Acknowledgements}

This paper reports the conclusions of the second MRI-Path Workshop, which was held in Milan, Italy, Nov 23-24, 2017, and was chaired by Massimo Filippi and Wolfgang Brück. The workshop was supported by an unrestricted education grant from Merck-Serono. The funding source had no role in the preparation of this article. We are very grateful to D.L. Arnold (McGill University, Montreal, Canada; Montreal Neurological Institute and Hospital, Department of Neurology and Neurosurgery, Montreal, Canada) for his fruitful discussion during the meeting and his subsequent thoughtful comments on the manuscript. 


\section{Conflict of interest statement}

M. Filippi is Editor-in-Chief of the Journal of Neurology; received compensation for consulting services and/or speaking activities from Biogen Idec, Merck-Serono, Novartis, Teva Pharmaceutical Industries; and receives research support from Biogen Idec, Merck-Serono, Novartis, Teva Pharmaceutical Industries, Roche, Italian Ministry of Health, Fondazione Italiana Sclerosi Multipla, and ARiSLA (Fondazione Italiana di Ricerca per la SLA).

W. Brück served on the scientific advisory board of Genzyme, Novartis, Biogen, and Teva Pharma; received speaker honoraria from Teva, Sanofi, Genzyme, Novartis, Merck Serono, Biogen, Roche, and Bayer; is an editorial board member of Therapeutic Advances in Neurological Disorders and Multiple Sclerosis International and Neuropathology and Applied Neurobiology; and received research support from Teva Pharma, Novartis, Biogen, Genzyme, MedDay, the German Research Foundation, German Ministry for Education and Research, the Tschira Foundation, and the German Multiple Sclerosis Foundation.

D. Chard has had meeting expenses funded by Merck and Novartis. He has received research funding from the International Progressive MS Alliance, the MS Society of Great Britain and Northern Ireland, and the National Institute for Health Research (NIHR) University College London Hospitals (UCLH) Biomedical Research Centre.

F. Fazekas declares no competing interests.

J.J.G. Geurts serves on the editorial boards of MS Journal, BMC Neurology, MS International and Neurology and the Scientific Advisory Board of the Dutch MS Research Foundation and of MS Academia, Merck Serono, and has received personal fees for consultancy from Merck-Serono, Biogen Idec, Novartis, Sanofi Genzyme, and Teva Pharmaceuticals.

C. Enzinger received funding for traveling and speaker honoraria from Biogen Idec, Bayer Schering Pharma, Merck Serono, Novartis, Genzyme and Teva Pharmaceutical Industries Ltd./sanofiAventis; received research support from Merck Serono, Biogen Idec, and Teva Pharmaceutical Industries Ltd./sanofi-Aventis; and serves on scientific advisory boards for Bayer Schering Pharma, Biogen Idec, Merck Serono, Novartis, Genzyme, Roche, and Teva Pharmaceutical Industries Ltd./sanofi- Aventis.

S. Hametner declares no competing interests.

T. Kuhlmann received speaker honoraria from Novartis and EXCEMED. She receives funding from the SFB-TRR 128 (DFG), European Leukodystrophy Association, International Progressive MS Alliance and the Interdisciplinary Clinical Research Center Münster. She is an editorial board member of Acta Neuropathologica and Multiple Sclerosis Journal. P. Preziosa received speaker honoraria from Biogen Idec, Novartis, and EXCEMED. 
A. Rovira serves on scientific advisory boards for Biogen Idec, Novartis, Sanofi-Genzyme, Icometrix, SyntheticMR, and OLEA Medical and has received speaker honoraria from Roche, Bayer, Sanofi-Genzyme, Bracco, Merck-Serono, Teva Pharmaceutical Industries Ltd, Novartis, and Biogen Idec.

K. Schmierer reports being a principal investigator of trials sponsored by Biogen, Roche, Teva, and MedDay; involved in trials sponsored by Sanofi Genzyme, BIAL, Cytokinetics, and Canbex; and receiving speaking honoraria from Biogen, MSD, Merck Serono, Novartis, Roche, SanofiGenzyme, and Teva.

C. Stadelmann reports personal fees for lecturing and advisory activity from Novartis, Bayer, Merck, and Roche and grants from Teva. She also reports research support by the German Research Foundation (DFG), the Gemeinnützige Hertie-Stiftung, and the German Multiple Sclerosis Society (DMSG).

M.A. Rocca received speakers honoraria from Biogen Idec, Novartis, Genzyme, Sanofi-Aventis, Teva, Merck Serono, and Roche and receives research support from the Italian Ministry of Health and Fondazione Italiana Sclerosi Multipla. 
Authors' contribution. TK and AR drafted the section on WM lesions. JJG and PP drafted the section on GM lesions. KS and DC drafted the section on spinal cord. WB and CE drafted the section on normal-appearing brain tissue. CS and MAR drafted the section on critical structures. SH and FF drafted the section on iron pathology. MF drafted the introductory and concluding sections and merged the different sections into the complete manuscript, which was commented on, revised and approved additionally by all other coauthors. 


\section{References}

1. Filippi M, Rocca MA, Barkhof F, et al. Association between pathological and MRI findings in multiple sclerosis. Lancet Neurol 2012; 11(4): 349-60.

2. Kuhlmann T, Ludwin S, Prat A, Antel J, Bruck W, Lassmann H. An updated histological classification system for multiple sclerosis lesions. Acta Neuropathol 2017; 133(1): 13-24.

3. Frischer JM, Weigand SD, Guo Y, et al. Clinical and pathological insights into the dynamic nature of the white matter multiple sclerosis plaque. Ann Neurol 2015; 78(5): 710-21.

4. Lucchinetti C, Bruck W, Parisi J, Scheithauer B, Rodriguez M, Lassmann H. Heterogeneity of multiple sclerosis lesions: implications for the pathogenesis of demyelination. Ann Neurol 2000; 47(6): 707-17.

5. van der Goes A, Boorsma W, Hoekstra K, Montagne L, de Groot CJ, Dijkstra CD. Determination of the sequential degradation of myelin proteins by macrophages. J Neuroimmunol 2005; 161(1-2): 12-20.

6. Luchetti S, Fransen NL, van Eden CG, Ramaglia V, Mason M, Huitinga I. Progressive multiple sclerosis patients show substantial lesion activity that correlates with clinical disease severity and sex: a retrospective autopsy cohort analysis. Acta Neuropathol 2018; 135(4): 511-28.

7. Kuhlmann T, Lingfeld G, Bitsch A, Schuchardt J, Brück W. Acute axonal damage in multiple sclerosis is most extensive in early disease stages and decreases over time. Brain 2002; 125: $2202-12$.

8. Miron VE, Franklin RJ. Macrophages and CNS remyelination. J Neurochem 2014; 130(2): 165-71.

9. Goldschmidt T, Antel J, Konig FB, Bruck W, Kuhlmann T. Remyelination capacity of the MS brain decreases with disease chronicity. Neurology 2009; 72(22): 1914-21.

10. Franklin RJM, Ffrench-Constant C. Regenerating CNS myelin - from mechanisms to experimental medicines. Nat Rev Neurosci 2017; 18(12): 753-69.

11. Cantuti-Castelvetri L, Fitzner D, Bosch-Queralt M, et al. Defective cholesterol clearance limits remyelination in the aged central nervous system. Science 2018; 359(6376): 684-8.

12. Stangel M, Kuhlmann T, Matthews PM, Kilpatrick TJ. Achievements and obstacles of remyelinating therapies in multiple sclerosis. Nat Rev Neurol 2017; 13(12): 742-54.

13. Geraldes R, Ciccarelli O, Barkhof F, et al. The current role of MRI in differentiating multiple sclerosis from its imaging mimics. Nat Rev Neurol 2018; 14(4): 199-213.

14. Solomon AJ, Bourdette DN, Cross AH, et al. The contemporary spectrum of multiple sclerosis misdiagnosis: A multicenter study. Neurology 2016; 87(13): 1393-9.

15. Sati P, Oh J, Constable RT, et al. The central vein sign and its clinical evaluation for the diagnosis of multiple sclerosis: a consensus statement from the North American Imaging in Multiple Sclerosis Cooperative. Nat Rev Neurol 2016; 12(12): 714-22.

16. Campion T, Smith RJP, Altmann DR, et al. FLAIR* to visualize veins in white matter lesions: A new tool for the diagnosis of multiple sclerosis? Eur Radiol 2017; 27(10): 4257-63.

17. Kilsdonk ID, Wattjes MP, Lopez-Soriano A, et al. Improved differentiation between MS and vascular brain lesions using FLAIR* at 7 Tesla. Eur Radiol 2014; 24(4): 841-9.

18. Sinnecker T, Dorr J, Pfueller CF, et al. Distinct lesion morphology at 7-T MRI differentiates neuromyelitis optica from multiple sclerosis. Neurology 2012; 79(7): 708-14.

19. Solomon AJ, Schindler MK, Howard DB, et al. "Central vessel sign" on 3T FLAIR* MRI for the differentiation of multiple sclerosis from migraine. Ann Clin Transl Neurol 2016; 3(2): 82-7. 20. Sati P, George IC, Shea CD, Gaitan MI, Reich DS. FLAIR*: a combined MR contrast technique for visualizing white matter lesions and parenchymal veins. Radiology 2012; 265(3): 92632.

21. Maggi P, Absinta M, Grammatico M, et al. Central vein sign differentiates Multiple Sclerosis from central nervous system inflammatory vasculopathies. Ann Neurol 2018; 83(2): 28394. 
22. Cortese R, Magnollay L, Tur C, et al. Value of the central vein sign at $3 \mathrm{~T}$ to differentiate MS from seropositive NMOSD. Neurology 2018; 90(14): e1183-e90.

23. Dal-Bianco A, Grabner G, Kronnerwetter C, et al. Slow expansion of multiple sclerosis iron rim lesions: pathology and $7 \mathrm{~T}$ magnetic resonance imaging. Acta Neuropathol 2017; 133(1): 25-42. 24. Chen W, Gauthier SA, Gupta A, et al. Quantitative susceptibility mapping of multiple sclerosis lesions at various ages. Radiology 2014; 271(1): 183-92.

25. Absinta M, Sati P, Schindler M, et al. Persistent 7-tesla phase rim predicts poor outcome in new multiple sclerosis patient lesions. The Journal of clinical investigation 2016; 126(7): 2597-609. 26. Kutzelnigg A, Faber-Rod JC, Bauer J, et al. Widespread demyelination in the cerebellar cortex in multiple sclerosis. Brain Pathol 2007; 17(1): 38-44.

27. Vercellino M, Masera S, Lorenzatti M, et al. Demyelination, inflammation, and neurodegeneration in multiple sclerosis deep gray matter. J Neuropathol Exp Neurol 2009; 68(5): 489-502.

28. Geurts JJ, Bo L, Roosendaal SD, et al. Extensive hippocampal demyelination in multiple sclerosis. J Neuropathol Exp Neurol 2007; 66(9): 819-27.

29. Carassiti D, Altmann DR, Petrova N, Pakkenberg B, Scaravilli F, Schmierer K. Neuronal loss, demyelination and volume change in the multiple sclerosis neocortex. Neuropathol Appl Neurobiol 2018; 44(4): 377-90.

30. Petrova N, Carassiti D, Altmann DR, Baker D, Schmierer K. Axonal loss in the multiple sclerosis spinal cord revisited. Brain Pathol 2018; 28(3): 334-48.

31. Gilmore CP, Donaldson I, Bo L, Owens T, Lowe J, Evangelou N. Regional variations in the extent and pattern of grey matter demyelination in multiple sclerosis: a comparison between the cerebral cortex, cerebellar cortex, deep grey matter nuclei and the spinal cord. J Neurol Neurosurg Psychiatry 2009; 80(2): 182-7.

32. Howell OW, Schulz-Trieglaff EK, Carassiti D, et al. Extensive grey matter pathology in the cerebellum in multiple sclerosis is linked to inflammation in the subarachnoid space. Neuropathol Appl Neurobiol 2015; 41(6): 798-813.

33. Albert M, Barrantes-Freer A, Lohrberg M, et al. Synaptic pathology in the cerebellar dentate nucleus in chronic multiple sclerosis. Brain Pathol 2017; 27(6): 737-47.

34. Bo L, Vedeler CA, Nyland HI, Trapp BD, Mork SJ. Subpial demyelination in the cerebral cortex of multiple sclerosis patients. J Neuropathol Exp Neurol 2003; 62(7): 723-32.

35. Lucchinetti CF, Popescu BFG, Bunyan RF, et al. Inflammatory cortical demyelination in early multiple sclerosis. $N$ Engl J Med 2011; 365: 2188-97.

36. Magliozzi R, Howell OW, Reeves C, et al. A Gradient of neuronal loss and meningeal inflammation in multiple sclerosis. Ann Neurol 2010; 68(4): 477-93.

37. Lagumersindez-Denis N, Wrzos C, Mack M, et al. Differential contribution of immune effector mechanisms to cortical demyelination in multiple sclerosis. Acta Neuropathol 2017; 134(1): 15-34.

38. Watkins LM, Neal JW, Loveless S, et al. Complement is activated in progressive multiple sclerosis cortical grey matter lesions. J Neuroinflammation 2016; 13(1): 161.

39. Yates RL, Esiri MM, Palace J, Jacobs B, Perera R, DeLuca GC. Fibrin(ogen) and neurodegeneration in the progressive multiple sclerosis cortex. Ann Neurol 2017; 82(2): 259-70.

40. Kooi EJ, Strijbis EM, van der Valk P, Geurts JJ. Heterogeneity of cortical lesions in multiple sclerosis: clinical and pathologic implications. Neurology 2012; 79(13): 1369-76.

41. Herranz E, Gianni C, Louapre C, et al. Neuroinflammatory component of gray matter pathology in multiple sclerosis. Ann Neurol 2016; 80(5): 776-90.

42. Yates RL, Esiri MM, Palace J, Mittal A, DeLuca GC. The influence of HLA-DRB1*15 on motor cortical pathology in multiple sclerosis. Neuropathol Appl Neurobiol 2015; 41(3): 371-84.

43. Hendrickx DAE, van Scheppingen J, van der Poel M, et al. Gene Expression Profiling of Multiple Sclerosis Pathology Identifies Early Patterns of Demyelination Surrounding Chronic Active Lesions. Front Immunol 2017; 8: 1810. 
44. Absinta M, Vuolo L, Rao A, et al. Gadolinium-based MRI characterization of leptomeningeal inflammation in multiple sclerosis. Neurology 2015; 85(1): 18-28.

45. Absinta M, Cortese IC, Vuolo L, et al. Leptomeningeal gadolinium enhancement across the spectrum of chronic neuroinflammatory diseases. Neurology 2017; 88(15): 1439-44.

46. Popescu V, Klaver R, Voorn P, et al. What drives MRI-measured cortical atrophy in multiple sclerosis? Mult Scler 2015; 21(10): 1280-90.

47. Haider L, Simeonidou C, Steinberger G, et al. Multiple sclerosis deep grey matter: the relation between demyelination, neurodegeneration, inflammation and iron. J Neurol Neurosurg Psychiatry 2014; 85(12): 1386-95.

48. Jurgens T, Jafari M, Kreutzfeldt M, et al. Reconstruction of single cortical projection neurons reveals primary spine loss in multiple sclerosis. Brain 2016; 139(Pt 1): 39-46.

49. Strijbis EMM, Kooi EJ, van der Valk P, Geurts JJG. Cortical Remyelination Is Heterogeneous in Multiple Sclerosis. J Neuropathol Exp Neurol 2017; 76(5): 390-401.

50. Geurts JJ, Roosendaal SD, Calabrese M, et al. Consensus recommendations for MS cortical lesion scoring using double inversion recovery MRI. Neurology 2011; 76(5): 418-24.

51. Sethi V, Yousry TA, Muhlert N, et al. Improved detection of cortical MS lesions with phasesensitive inversion recovery MRI. J Neurol Neurosurg Psychiatry 2012; 83(9): 877-82.

52. Nelson F, Poonawalla A, Hou P, Wolinsky JS, Narayana PA. 3D MPRAGE improves classification of cortical lesions in multiple sclerosis. Mult Scler 2008; 14(9): 1214-9.

53. Seewann A, Kooi EJ, Roosendaal SD, et al. Postmortem verification of MS cortical lesion detection with 3D DIR. Neurology 2012; 78(5): 302-8.

54. Kilsdonk ID, Jonkman LE, Klaver R, et al. Increased cortical grey matter lesion detection in multiple sclerosis with 7 T MRI: a post-mortem verification study. Brain 2016; 139(5): 1472-81.

55. Mainero C, Benner T, Radding A, et al. In vivo imaging of cortical pathology in multiple sclerosis using ultra-high field MRI. Neurology 2009; 73(12): 941-8.

56. Preziosa P, Rocca MA, Mesaros S, et al. Diagnosis of multiple sclerosis: a multicentre study to compare revised McDonald-2010 and Filippi-2010 criteria. J Neurol Neurosurg Psychiatry 2018; 89(3): 316-8.

57. Absinta M, Rocca MA, Colombo B, et al. Patients with migraine do not have MRI-visible cortical lesions. J Neurol 2012; 259: 2695-8.

58. Calabrese M, Oh MS, Favaretto A, et al. No MRI evidence of cortical lesions in neuromyelitis optica. Neurology 2012; 79(16): 1671-6.

59. Filippi M, Rocca MA, Ciccarelli O, et al. MRI criteria for the diagnosis of multiple sclerosis: MAGNIMS consensus guidelines. Lancet Neurol 2016; 15(3): 292-303.

60. Scalfari A, Romualdi C, Nicholas RS, et al. The cortical damage, early relapses, and onset of the progressive phase in multiple sclerosis. Neurology 2018; 90(24): e2197-e18.

61. Chen JT, Easley K, Schneider C, et al. Clinically feasible MTR is sensitive to cortical demyelination in MS. Neurology 2013; 80(3): 246-52.

62. Schmierer K, Parkes HG, So PW, et al. High field (9.4 Tesla) magnetic resonance imaging of cortical grey matter lesions in multiple sclerosis. Brain 2010; 133(Pt 3): 858-67.

63. Derakhshan M, Caramanos Z, Narayanan S, Arnold DL, Louis Collins D. Surface-based analysis reveals regions of reduced cortical magnetization transfer ratio in patients with multiple sclerosis: a proposed method for imaging subpial demyelination. Hum Brain Mapp 2014; 35(7): 3402-13.

64. Samson RS, Cardoso MJ, Muhlert N, et al. Investigation of outer cortical magnetisation transfer ratio abnormalities in multiple sclerosis clinical subgroups. Mult Scler 2014; 20(10): 132230.

65. Filippi M, Preziosa P, Pagani E, et al. Microstructural MR imaging of cortical lesion in multiple sclerosis. Mult Scler 2013; 19(4): 418-26.

66. Preziosa P, Pagani E, Morelli ME, et al. DT MRI microstructural cortical lesion damage does not explain cognitive impairment in MS. Mult Scler 2017; 23(14): 1918-28. 
67. Granberg T, Fan Q, Treaba CA, et al. In vivo characterization of cortical and white matter neuroaxonal pathology in early multiple sclerosis. Brain 2017; 140(11): 2912-26.

68. Yaldizli O, Pardini M, Sethi V, et al. Characteristics of lesional and extra-lesional cortical grey matter in relapsing-remitting and secondary progressive multiple sclerosis: A magnetisation transfer and diffusion tensor imaging study. Mult Scler 2016; 22(2): 150-9.

69. Jonkman LE, Klaver R, Fleysher L, Inglese M, Geurts JJ. The substrate of increased cortical FA in MS: A 7T post-mortem MRI and histopathology study. Mult Scler 2016; 22(14): 1804-11. 70. Nakamura K, Chen JT, Ontaneda D, Fox RJ, Trapp BD. T1-/T2-weighted ratio differs in demyelinated cortex in multiple sclerosis. Ann Neurol 2017; 82(4): 635-9.

71. Righart R, Biberacher V, Jonkman LE, et al. Cortical pathology in multiple sclerosis detected by the T1/T2-weighted ratio from routine magnetic resonance imaging. Ann Neurol 2017; 82(4): 519-29.

72. Bjartmar C, Kidd G, Mork S, Rudick R, Trapp BD. Neurological disability correlates with spinal cord axonal loss and reduced $\mathrm{N}$-acetyl aspartate in chronic multiple sclerosis patients. Ann Neurol 2000; 48(6): 893-901.

73. Uchida Y, Sumiya T, Tachikawa M, et al. Involvement of Claudin-11 in Disruption of Blood-Brain, -Spinal Cord, and -Arachnoid Barriers in Multiple Sclerosis. Mol Neurobiol 2018.

74. Gilmore CP, Geurts JJ, Evangelou N, et al. Spinal cord grey matter lesions in multiple sclerosis detected by post-mortem high field MR imaging. Mult Scler 2009; 15(2): 180-8.

75. Thompson AJ, Banwell BL, Barkhof F, et al. Diagnosis of multiple sclerosis: 2017 revisions of the McDonald criteria. Lancet Neurol 2018; 17(2): 162-73.

76. Rocca MA, Horsfield MA, Sala S, et al. A multicenter assessment of cervical cord atrophy among MS clinical phenotypes. Neurology 2011; 76(24): 2096-102.

77. Montalban X, Sastre-Garriga J, Tintore M, et al. A single-center, randomized, double-blind, placebo-controlled study of interferon beta- $1 \mathrm{~b}$ on primary progressive and transitional multiple sclerosis. Mult Scler 2009; 15(10): 1195-205.

78. Kapoor R, Furby J, Hayton T, et al. Lamotrigine for neuroprotection in secondary progressive multiple sclerosis: a randomised, double-blind, placebo-controlled, parallel-group trial. Lancet Neurol 2010; 9(7): 681-8.

79. Singhal T, Tauhid S, Hurwitz S, Neema M, Bakshi R. The Effect of Glatiramer Acetate on Spinal Cord Volume in Relapsing-Remitting Multiple Sclerosis. J Neuroimaging 2017; 27(1): 33-6.

80. Kearney H, Rocca MA, Valsasina P, et al. Magnetic resonance imaging correlates of physical disability in relapse onset multiple sclerosis of long disease duration. Mult Scler 2014; 20(1): 72-80.

81. Schlaeger R, Papinutto N, Panara V, et al. Spinal cord gray matter atrophy correlates with multiple sclerosis disability. Ann Neurol 2014; 76(4): 568-80.

82. Schmierer K, Wheeler-Kingshott C, Tozer DJ, et al. Quantitative Magnetic Resonance of Postmortem Multiple Sclerosis Brain before and after Fixation. Magnetic Resonance in Medicine: Official Journal of the Society of Magnetic Resonance in Medicine / Society of Magnetic Resonance in Medicine 2008; 59(2): 268-77.

83. Wang Y, Sun P, Wang Q, et al. Differentiation and quantification of inflammation, demyelination and axon injury or loss in multiple sclerosis. Brain 2015; 138(Pt 5): 1223-38.

84. Wang Y, Wang Q, Haldar JP, et al. Quantification of increased cellularity during inflammatory demyelination. Brain 2011; 134(Pt 12): 3590-601.

85. Grussu F, Schneider T, Tur C, et al. Neurite dispersion: a new marker of multiple sclerosis spinal cord pathology? Ann Clin Transl Neurol 2017; 4(9): 663-79.

86. Grussu F, Schneider T, Yates RL, et al. A framework for optimal whole-sample histological quantification of neurite orientation dispersion in the human spinal cord. J Neurosci Methods 2016; 273: 20-32. 
87. Grussu F, Schneider T, Zhang H, Alexander DC, Wheeler-Kingshott CA. Neurite orientation dispersion and density imaging of the healthy cervical spinal cord in vivo. Neuroimage 2015; 111: 590-601.

88. Allen IV, McKeown SR. A histological, histochemical and biochemical study of the macroscopically normal white matter in multiple sclerosis. J Neurol Sci 1979; 41(1): 81-91. 89. Enzinger C, Barkhof F, Ciccarelli O, et al. Nonconventional MRI and microstructural cerebral changes in multiple sclerosis. Nat Rev Neurol 2015; 11(12): 676-86.

90. Moll NM, Rietsch AM, Thomas S, et al. Multiple sclerosis normal-appearing white matter: Pathology-imaging correlations. Ann Neurol 2011; 70: 764-73.

91. Jehna M, Pirpamer L, Khalil M, et al. Periventricular lesions correlate with cortical thinning in multiple sclerosis. Ann Neurol 2015; 78(4): 530-9.

92. Pardini M, Sudre $\mathrm{CH}$, Prados F, et al. Relationship of grey and white matter abnormalities with distance from the surface of the brain in multiple sclerosis. J Neurol Neurosurg Psychiatry 2016; 87(11): 1212-7.

93. Mainero C, Louapre C, Govindarajan ST, et al. A gradient in cortical pathology in multiple sclerosis by in vivo quantitative $7 \mathrm{~T}$ imaging. Brain 2015; 138(Pt 4): 932-45.

94. Vavasour IM, Huijskens SC, Li DK, et al. Global loss of myelin water over 5 years in multiple sclerosis normal-appearing white matter. Mult Scler 2017: 1352458517723717.

95. Minagar A, Barnett MH, Benedict RH, et al. The thalamus and multiple sclerosis: modern views on pathologic, imaging, and clinical aspects. Neurology 2013; 80(2): 210-9.

96. Parmar K, Stadelmann C, Rocca MA, et al. The role of the cerebellum in multiple sclerosis150 years after Charcot. Neurosci Biobehav Rev 2018; 89: 85-98.

97. Rocca MA, Barkhof F, De Luca J, et al. The hippocampus in multiple sclerosis. Lancet Neurol 2018; 17(10): 918-26.

98. Cifelli A, Arridge M, Jezzard P, Esiri MM, Palace J, Matthews PM. Thalamic neurodegeneration in multiple sclerosis. Ann Neurol 2002; 52(5): 650-3.

99. Redondo J, Kemp K, Hares K, Rice C, Scolding N, Wilkins A. Purkinje Cell Pathology and Loss in Multiple Sclerosis Cerebellum. Brain Pathol 2015; 25(6): 692-700.

100. Papadopoulos D, Dukes S, Patel R, Nicholas R, Vora A, Reynolds R. Substantial archaeocortical atrophy and neuronal loss in multiple sclerosis. Brain Pathol 2009; 19(2): 238-53.

101. Kooi EJ, Prins M, Bajic N, et al. Cholinergic imbalance in the multiple sclerosis hippocampus. Acta Neuropathol 2011; 122(3): 313-22.

102. Roosendaal SD, Moraal B, Pouwels PJ, et al. Accumulation of cortical lesions in MS: relation with cognitive impairment. Mult Scler 2009; 15(6): 708-14.

103. Preziosa P, Rocca MA, Mesaros S, et al. Relationship between damage to the cerebellar peduncles and clinical disability in multiple sclerosis. Radiology 2014; 271(3): 822-30.

104. Tovar-Moll F, Evangelou IE, Chiu AW, et al. Thalamic involvement and its impact on clinical disability in patients with multiple sclerosis: a diffusion tensor imaging study at $3 \mathrm{~T}$. AJNR Am J Neuroradiol 2009; 30(7): 1380-6.

105. Prosperini L, Sbardella E, Raz E, et al. Multiple sclerosis: white and gray matter damage associated with balance deficit detected at static posturography. Radiology 2013; 268(1): 181-9. 106. Planche V, Ruet A, Coupe P, et al. Hippocampal microstructural damage correlates with memory impairment in clinically isolated syndrome suggestive of multiple sclerosis. Mult Scler 2017; 23(9): 1214-24.

107. Mesaros S, Rocca MA, Pagani E, et al. Thalamic Damage Predicts the Evolution of Primary-Progressive Multiple Sclerosis at 5 Years. AJNR Am J Neuroradiol 2011; 32(6): 1016-20. 108. Sicotte NL, Kern KC, Giesser BS, et al. Regional hippocampal atrophy in multiple sclerosis. Brain 2008; 131(Pt 4): 1134-41.

109. Longoni G, Rocca MA, Pagani E, et al. Deficits in memory and visuospatial learning correlate with regional hippocampal atrophy in MS. Brain Struct Funct 2015; 220: 435-44. 
110. D'Ambrosio A, Pagani E, Riccitelli GC, et al. Cerebellar contribution to motor and cognitive performance in multiple sclerosis: An MRI sub-regional volumetric analysis. Mult Scler 2017; 23(9): 1194-203.

111. Bisecco A, Rocca MA, Pagani E, et al. Connectivity-based parcellation of the thalamus in multiple sclerosis and its implications for cognitive impairment: A multicenter study. Hum Brain Mapp 2015; 36(7): 2809-25.

112. Rocca MA, Pravata E, Valsasina P, et al. Hippocampal-DMN disconnectivity in MS is related to WM lesions and depression. Hum Brain Mapp 2015; 36(12): 5051-63.

113. Tona F, Petsas N, Sbardella E, et al. Multiple sclerosis: altered thalamic resting-state functional connectivity and its effect on cognitive function. Radiology 2014; 271(3): 814-21.

114. Cerasa A, Passamonti L, Valentino P, et al. Cerebellar-parietal dysfunctions in multiple sclerosis patients with cerebellar signs. Exp Neurol 2012; 237(2): 418-26.

115. Craelius W, Migdal MW, Luessenhop CP, Sugar A, Mihalakis I. Iron deposits surrounding multiple sclerosis plaques. Arch Pathol Lab Med 1982; 106(8): 397-9.

116. Drayer B, Burger P, Darwin R, Riederer S, Herfkens R, Johnson GA. MRI of brain iron. AJR Am J Roentgenol 1986; 147(1): 103-10.

117. Drayer B, Burger P, Hurwitz B, Dawson D, Cain J. Reduced signal intensity on MR images of thalamus and putamen in multiple sclerosis: increased iron content? AJR Am J Roentgenol 1987; 149(2): 357-63.

118. Bakshi R, Benedict RH, Bermel RA, et al. T2 hypointensity in the deep gray matter of patients with multiple sclerosis: a quantitative magnetic resonance imaging study. Arch Neurol 2002; 59(1): 62-8.

119. Walsh AJ, Lebel RM, Eissa A, et al. Multiple sclerosis: validation of MR imaging for quantification and detection of iron. Radiology 2013; 267(2): 531-42.

120. Langkammer C, Schweser F, Krebs N, et al. Quantitative susceptibility mapping (QSM) as a means to measure brain iron? A post mortem validation study. Neuroimage 2012; 62(3): 1593-9.

121. Ropele S, Enzinger C, Fazekas F. Iron Mapping in Multiple Sclerosis. Neuroimaging Clin N Am 2017; 27(2): 335-42.

122. Hametner S, Wimmer I, Haider L, Pfeifenbring S, Bruck W, Lassmann H. Iron and neurodegeneration in the multiple sclerosis brain. Ann Neurol 2013; 74(6): 848-61.

123. Mahad DH, Trapp BD, Lassmann H. Pathological mechanisms in progressive multiple sclerosis. Lancet Neurol 2015; 14(2): 183-93.

124. Ropele S, Kilsdonk ID, Wattjes MP, et al. Determinants of iron accumulation in deep grey matter of multiple sclerosis patients. Mult Scler 2014; 20(13): 1692-8.

125. Walsh AJ, Blevins G, Lebel RM, Seres P, Emery DJ, Wilman AH. Longitudinal MR imaging of iron in multiple sclerosis: an imaging marker of disease. Radiology 2014; 270(1): 18696.

126. Khalil M, Langkammer C, Pichler A, et al. Dynamics of brain iron levels in multiple sclerosis: A longitudinal 3T MRI study. Neurology 2015; 84(24): 2396-402.

127. Schweser F, Raffaini Duarte Martins AL, Hagemeier J, et al. Mapping of thalamic magnetic susceptibility in multiple sclerosis indicates decreasing iron with disease duration: A proposed mechanistic relationship between inflammation and oligodendrocyte vitality. Neuroimage 2018; 167: 438-52.

128. Popescu BF, Frischer JM, Webb SM, et al. Pathogenic implications of distinct patterns of iron and zinc in chronic MS lesions. Acta Neuropathol 2017; 134(1): 45-64.

129. Yao B, Hametner S, van Gelderen P, et al. 7 Tesla magnetic resonance imaging to detect cortical pathology in multiple sclerosis. PLoS One 2014; 9(10): e108863.

130. Zivadinov R, Ramasamy DP, Benedict RR, et al. Cerebral Microbleeds in Multiple Sclerosis Evaluated on Susceptibility-weighted Images and Quantitative Susceptibility Maps: A Case-Control Study. Radiology 2016; 281(3): 884-95. 
131. Birkl C, Carassiti D, Hussain F, et al. Assessment of ferritin content in multiple sclerosis brains using temperature-induced R*2 changes. Magn Reson Med 2018; 79(3): 1609-15. 


\section{Figure legends}

\section{Figure 1. Pathology of white matter lesions in patients with multiple sclerosis.}

Active lesions are characterized by complete demyelination (A) and numerous phagocytes throughout the lesion (B). Mixed active/inactive lesions are completely demyelinated (C), but have a rim of phagocytes at the lesion border (D). In contrast, demyelinated inactive lesions (E) have only a few scattered phagocytes within the lesions $(\mathrm{F})$. Note the presence of concentrated phagocytes in the myelinated normal appearing WM (arrow). Remyelination (small arrows) may be limited to the lesion border $(\mathrm{G})$ or present through the whole lesion $(\mathrm{H})$. Note the demyelinated cortex ( $\mathrm{G}$, big arrow) and a second demyelinated lesion (H, big arrow). The insert $(\mathrm{H})$ shows thin, irregularly formed remyelinated myelin sheaths. In A, C E, G and $\mathrm{H}$ immunohistochemistry for the myelin protein MBP. In B, D and F immunohistochemistry for CD68 which is expressed in blood derived monocytes and microglia. Scale bar in A: $500 \mu \mathrm{m}$; scale bar in insert in H: $200 \mu \mathrm{m}$.

\section{Figure 2. MRI of white matter lesions in patients with multiple sclerosis.}

(a-c) 3T brain MRI scan in a 32-year-old woman presenting with a clinically isolated syndrome (CIS) suggestive of MS showing a typical demyelinating ovoid lesion of the type seen in multiple sclerosis on a $T_{2}$-FLAIR image, involving the frontal periventricular white matter. Note the central vein on the susceptibility-weighted image (b) and on the combined $\mathrm{T}_{2}$-FLAIR and SWI $\left(\mathrm{T}_{2^{-}}\right.$ FLAIR*) images (arrows in b and c). (d-e) 3T brain MRI scan in a 34-year-old woman presenting with a CIS suggestive of MS, showing typical demyelinating lesions of the type seen in multiple sclerosis on a $\mathrm{T}_{2}$-FLAIR image (d), involving the periventricular white matter. Note the hypointense rims on the susceptibility-weighted images (e) in several of these lesions that likely reflect iron deposition (arrows). (f-g) Serial 3T brain MRI scan in a 56-year-old female with secondary progressive MS acquired at baseline (f), and two (g) and four (h) years later. Un-enhanced $\mathrm{T}_{1^{-}}$ 
weighted images demonstrate a slowly expanding lesion (with both size and hypointensity increase) in the left posterior periventricular white matter (arrows).

Figure 3. Cross-sectional analysis of the spinal cord of a patient with multiple sclerosis. Location of counting fields (red) randomly distributed over the area of the cortico-spinal tract (outline in blue) (A). Microscopy of one counting field (size: 120 x $120 \mu \mathrm{m}$ ) in normal (B-I) and MS (B-II) spinal cord. Scale bar: $50 \mu \mathrm{m}$. Magnified counting frames converted into black \& white (8 bit) of control (B-III) and MS (B-IV) cortico-spinal tract. Scale bar: $25 \mu \mathrm{m}$. All sections were immuno-stained for SMI31. Adapted from ${ }^{30}$ with permission.

Figure 4. In vivo MRI $\mathbf{R}_{2}{ }^{*}$ mapping and post mortem iron staining in patients with multiple sclerosis and healthy controls.

(a, b) Axial $\mathrm{R}_{2}{ }^{*}$ maps of (a) a 37-year-old healthy control and (b) a 33-year-old patient with early multiple sclerosis (MS) (Expanded Disability Status Scale score of 3.0). Note the increased $\mathrm{R}_{2}{ }^{*}$ relaxation rate in the basal ganglia, especially of the globus pallidus of the MS patient, as indicated by a higher intensity. (c, d) Coronal double-hemispheric histological sections of (c) a 63-year-old healthy control and (d) a 74-year-old patient with secondary progressive MS (Expanded Disability Status Scale score of 7.0), stained with the diaminobenzidine-enhanced Turnbull blue iron staining. Note the high iron density in the basal ganglia of the MS case, while iron appears depleted across the normal-appearing WM. A periventricular chronic MS lesion is outlined and magnified in the inset. The edge of the lesion is partially accentuated with iron accumulation in macrophages, forming an iron rim (blue arrows). Scale bar in (d): $500 \mu \mathrm{m}$. 
active lesion mixed active/inactive lesion

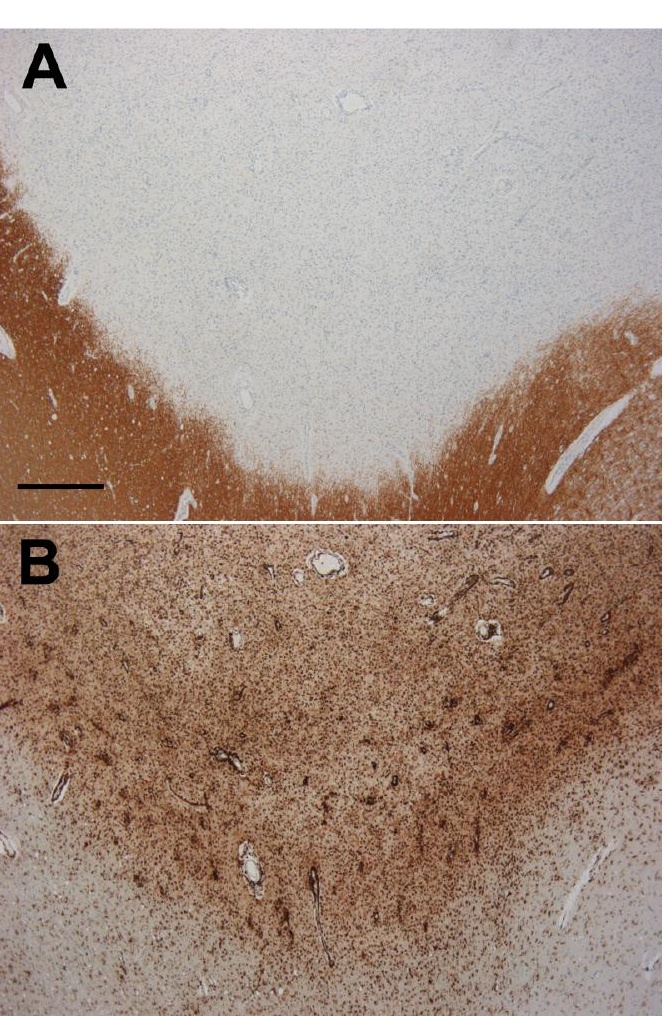

inactive

remyelination lesion

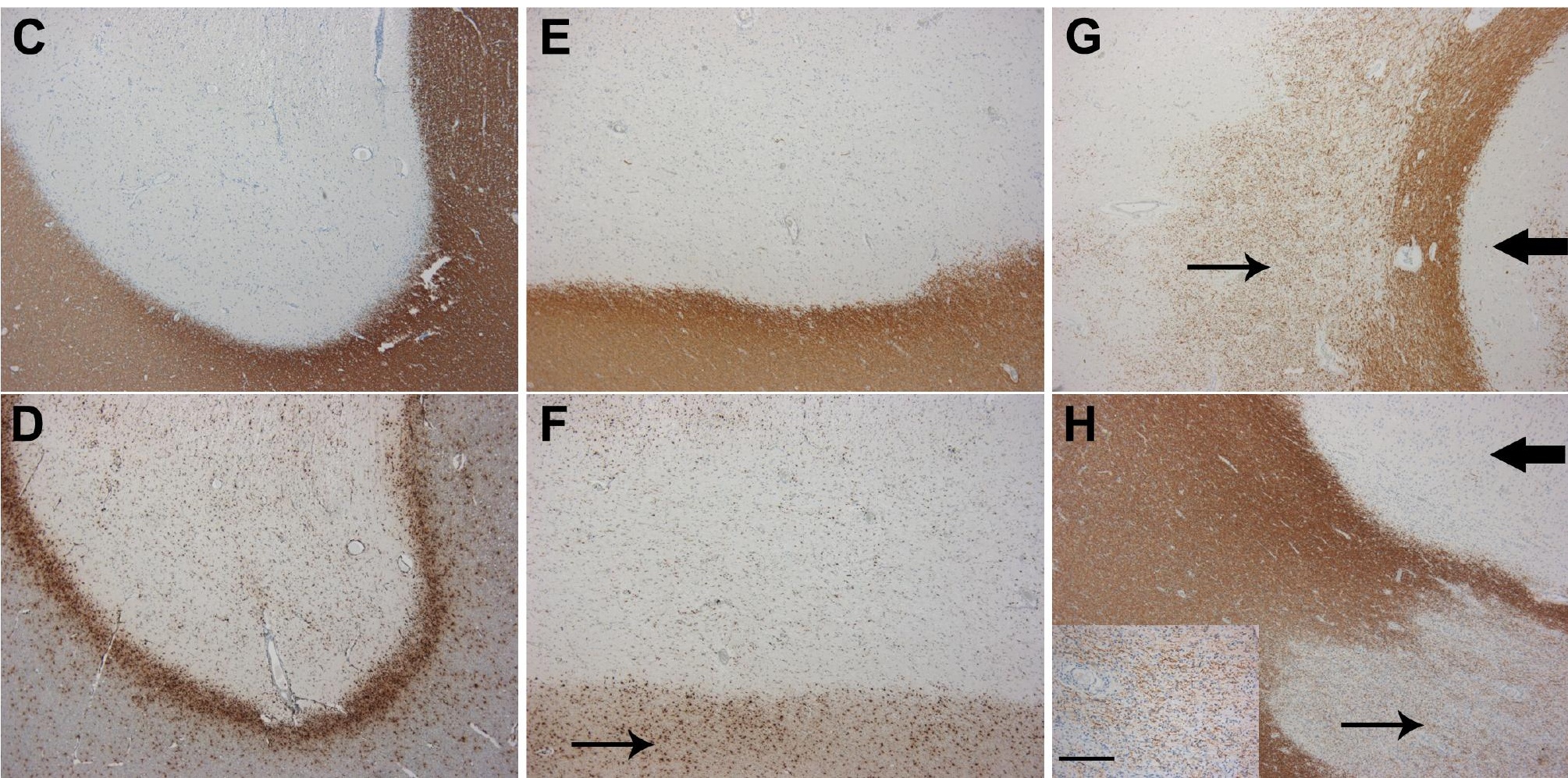



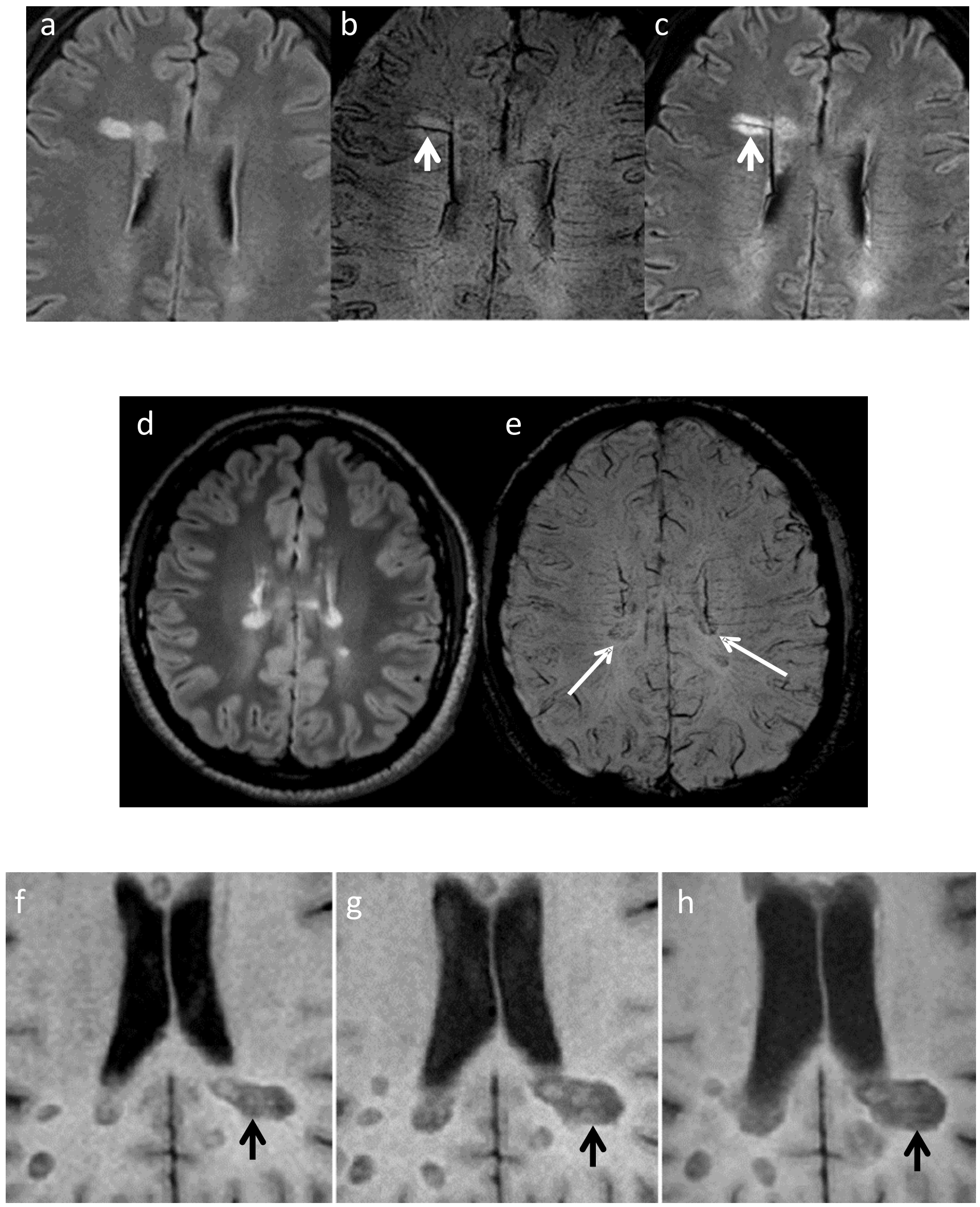

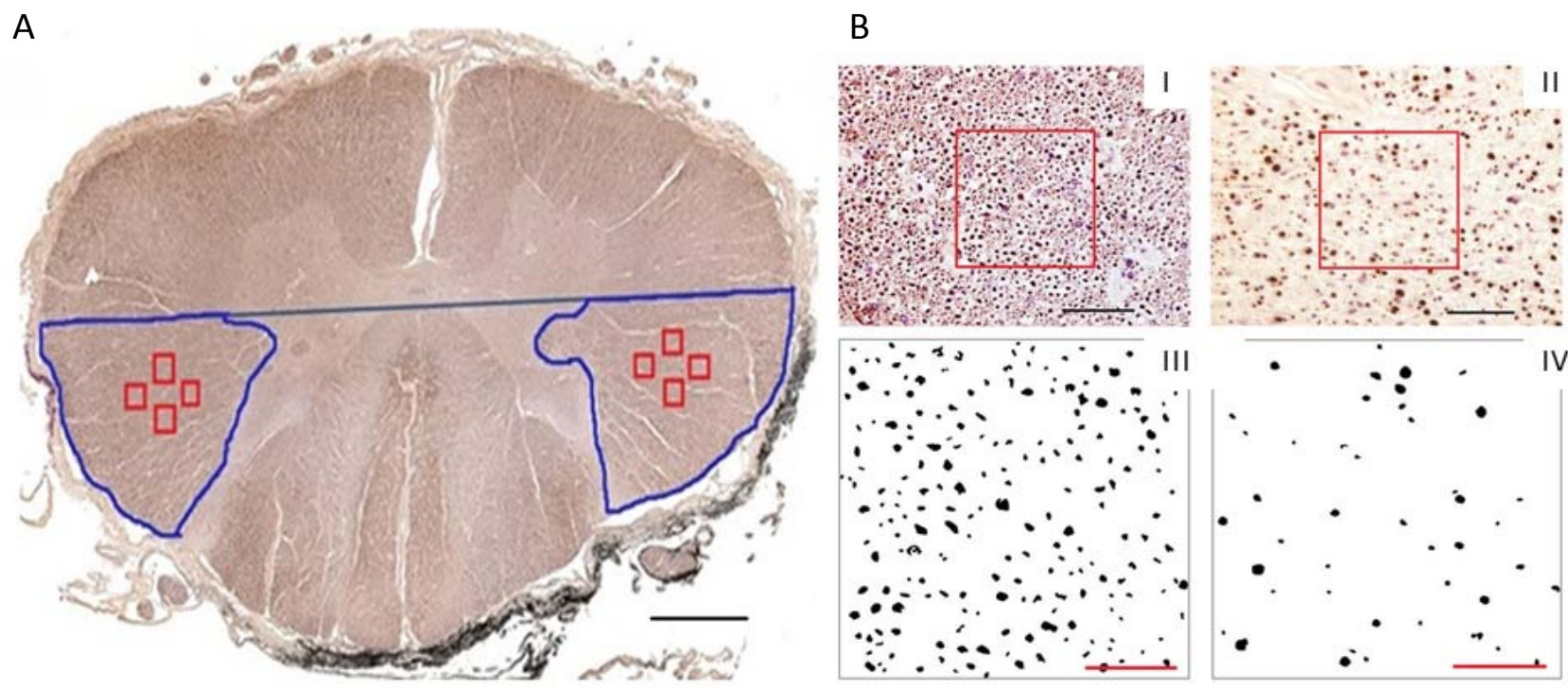


\section{Control}

a 2

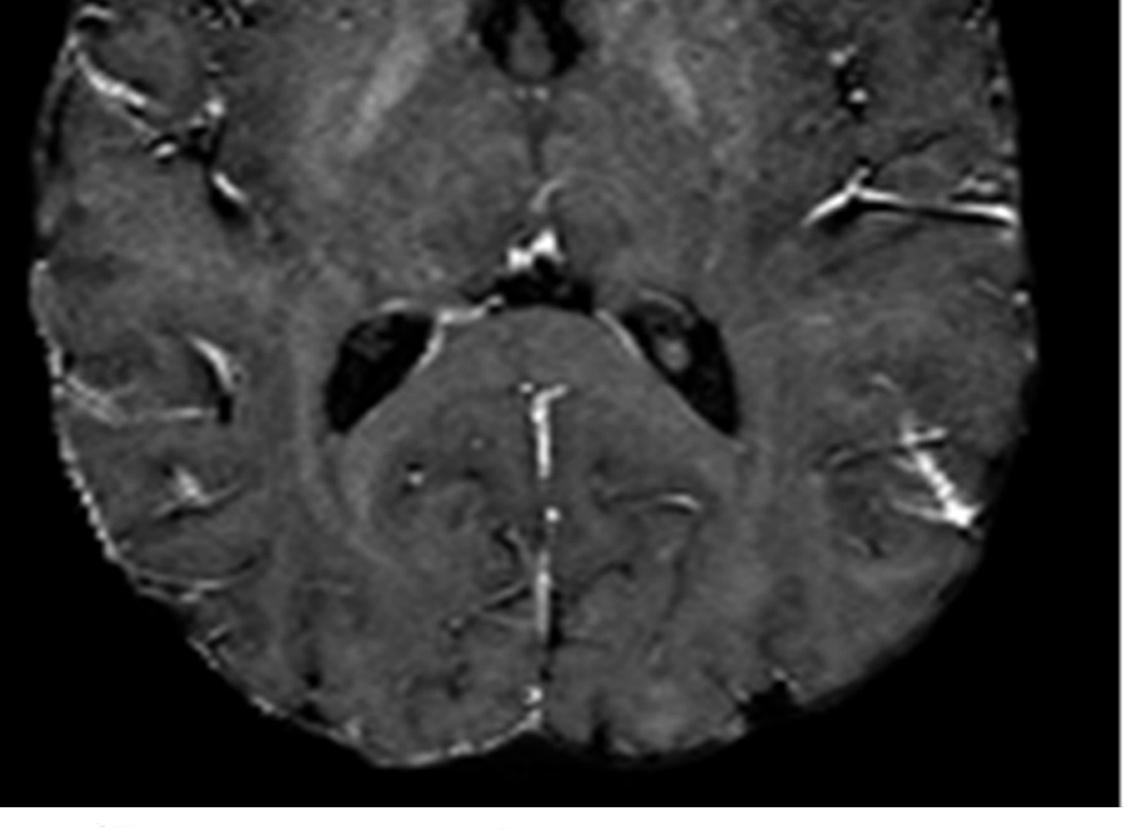

\section{C}

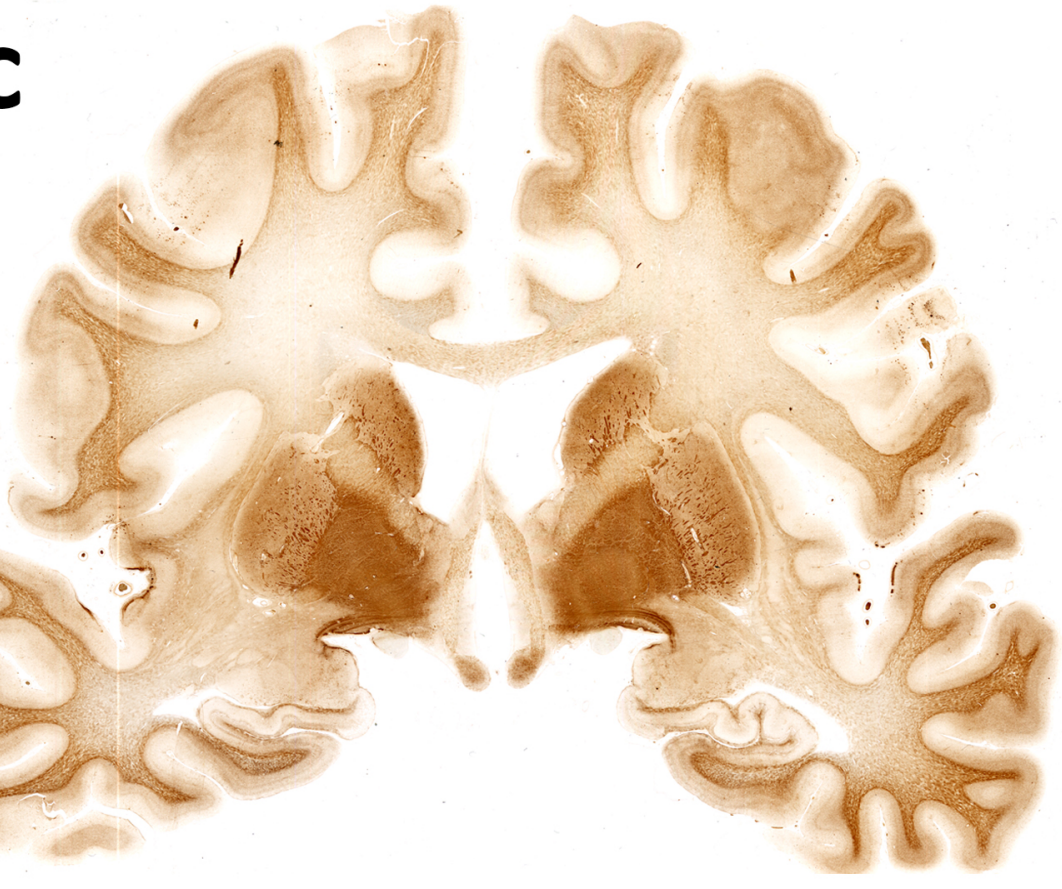

Multiple sclerosis
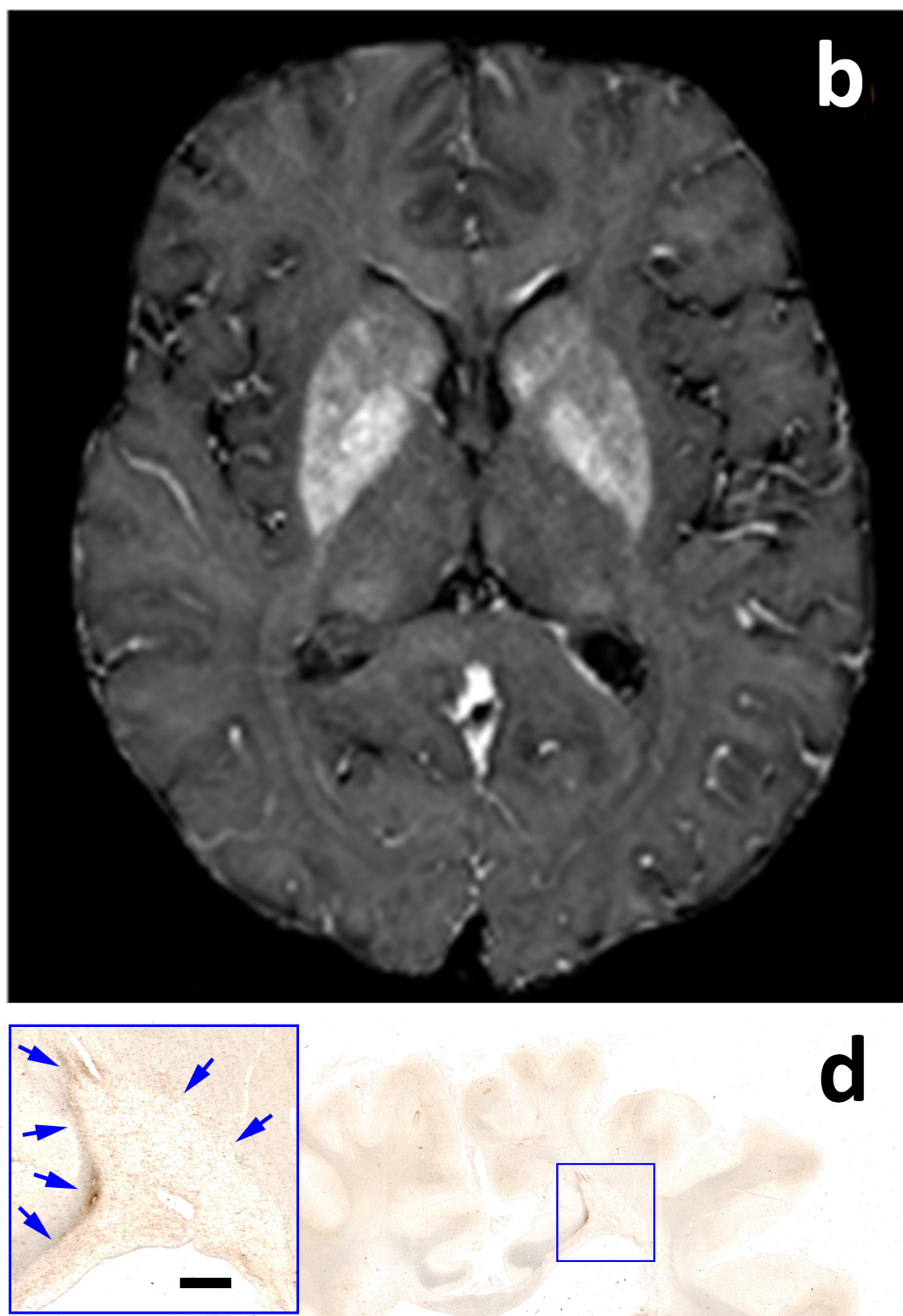

d 